Zugleich aber haben die Gerichte stets darauf bestanden, dass die Wehrdienstausnahmen gesetzlich festgelegt sein sollen und nicht beliebig von der Verwaltung ausgedehnt werden dürfen. ${ }^{15}$

Diese Rechtsprechung trifft jedoch nicht den Auswahlwehrdienst. Denn dieser statuiert keineswegs eine Befreiung von der Wehrpflicht, sondern nur eine an dem aktuellen Bedarf der Streitkräfte orientierte Nichtinanspruchnahme; sollte der Bedarf sich ändern, etwa bei einer Anspannung der Sicherheitslage, bleibt der Zugriff auf die betroffenen Wehrpflichtigen möglich. Zum anderen wird auch von denen, die den Grundsatz gesetzmäßiger Verwaltung zur Bedingung rechtmäßiger Einberufungspraxis machen, der Verwaltung zugestanden, dass sie etwa bei Wehrpflichtigen, die einer geringen Tauglichkeitsgruppe angehören, auch ohne zusätzliche Gesetzesregelung auf die Einberufung verzichtet: „Bei dem Tauglichkeitsgrad handelt es sich nämlich um ein Kriterium, das auf die Anforderungen der Armee zugeschnitten ist. "16 Da beim Auswahlwehrdienst

15 Dazu Voland op.cit. S. 455

16 Ebenda. gerade dies die Richtschnur ist, kann mangelnde Gesetzmäßigkeit der Verwaltung, falls kein willkürliches Handeln vorliegt, nicht gegen seine Rechtmäßigkeit angeführt werden.

\section{Zusammenfassung}

Die Zweifel an der Verfassungsmäßigkeit des Auswahlwehrdienstes in der Bundeswehr sind somit nur in einem Punkt berechtigt. Wenn nur noch ein kleiner Anteil der tauglich gemusterten Wehrpflichtigen für den Bedarf der Streitkräfte ausreicht, stellt der Wehrdienst keine Freiheitseinschränkung für alle Wehrpflichtigen, sondern nur noch für eine Minderheit dar. Ihr Sonderopfer muss ausgeglichen werden, am sinnvollsten durch eine angemessene Besoldung. Dies sollte eine künftige Entscheidung über die Verfassungsmäßigkeit der allgemeinen Wehrpflicht leiten. Und das Bundesverteidigungsministerium sollte nicht erst vom Verfassungsgericht veranlasst werden, den Auswahlwehrdienst vom Verdacht der Verfassungswidrigkeit zu befreien.

\title{
Eine Auswahlwehrpflicht greift zu kurz!
}

\author{
Jürgen Groß*
}

$\mathrm{D}$ ie Einführung einer „Auswahlwehrpflicht" war eine Empfehlung der sogenannten „WeizsäckerKommission", die von der rot-grünen Bundesregierung im Jahr 1999 eingerichtet wurde, um endlich ein schlüssiges Gesamtkonzept für die überfälligen Reformen in der Bundeswehr, die von der Vorgängerregierung fast ein Jahrzehnt lang verschleppt worden waren, zu erarbeiten.

Diese Empfehlung war zweifellos ein zentrales, innovatives Element in dem Bericht, den die Kommission im Mai 2000 vorlegte. ${ }^{1}$ Sie teilte ihr Schicksal mit einer Reihe weiterer zentraler, innovativer Elemente, die ebenfalls darin enthalten waren: Sie wurden von der Bundesregierung allesamt nicht aufgegriffen. Die Arbeit der Kommission wurde zwar gelobt, aber weitgehend ignoriert. Die vollmundig angekündigte „Erneuerung von Grund auf " ${ }^{\prime 2}$ fand nicht statt. Die Bundeswehr krankt daran bis heute.

Indes: Das Bedauern darüber, dass der Vorschlag, eine Auswahlwehrpflicht einzuführen, nicht realisiert wurde, muss sich deshalb in Grenzen halten, weil diese Neuerung einerseits

* Dr. Jürgen Groß ist Geschäftsführender Vorsitzender der Kommission „Europäische Sicherheit und Zukunft der Bundeswehr" am IFSH.

1 Gemeinsame Sicherheit und Zukunft der Bundeswehr. Bericht der Kommission an die Bundesregierung, 23. Mai 2000.

2 Der Bundesminister der Verteidigung, Die Bundeswehr - sicher ins 21. Jahrhundert. Eckpfeiler für eine Erneuerung von Grund auf, o.J. (2000). zwar gegenüber der bis zum heutigen Tag praktizierten Einberufungspraxis in den deutschen Streitkräften eine wirkliche Verbesserung bedeutet hätte, andererseits aber keineswegs die fortschrittlichste Idee war, die die erwähnte Kommission hinsichtlich der Wehrform diskutiert hat. Eine Minderheit der Kommissionsmitglieder war nämlich schon damals bereit, einen Schritt weiterzugehen und auf die Wehrpflicht ganz zu verzichten. Die Mehrzahl lehnte dies, mit keineswegs überzeugenden Argumenten übrigens, jedoch $\mathrm{ab}^{3}$ - ebenso, wie der seinerzeit amtierende Verteidigungsminister dann das Mehrheitsvotum für die Auswahlwehrpflicht ablehnte. (Angesichts dieser Abläufe könnte man fast geneigt sein zu sagen, dass es manchmal eben doch noch Gerechtigkeit auf der Welt gäbe wenn die Bundeswehr und vor allem die Hunderttausende, die infolgedessen weiter einberufen wurden, dabei nicht den Schaden gehabt hätten.)

Die Kernfrage, die sich nach dem Ende des Kalten Krieges in Europa (und damit auch dem Ende der Notwendigkeit, Massenheere zu unterhalten) geradezu aufdrängte, war doch nicht, auf welche Weise die allgemeine Wehrpflicht "weiterzuentwickeln" sei, ${ }^{4}$ sondern ob eben diese Wehrpflicht in Anbetracht

3 Vgl. Gemeinsame Sicherheit, a.a.O., S. 60, 68f.

4 Christian Bernzen/Christoph Bertram, Auswahlwehrpflicht: eine zeitgemäße Weiterentwicklung der allgemeinen Wehrpflicht? in diesem Heft. 
der fundamentalen Veränderung der sicherheitspolitischen Lage nicht ganz abgeschafft werden könne. Und selbst wenn vor zehn Jahren vielleicht noch irgendwelche Restzweifel eine gewisse Berechtigung hatten - heute liegt die Antwort nun wirklich für jedermann klar auf der Hand.

Folgerichtig sind in der Zwischenzeit fast alle Partner und Verbündeten der Bundesrepublik von ihren Wehrpflichtigenarmeen zu Freiwilligenstreitkräften übergegangen und, alles in allem, auch gut damit gefahren - was man schon daraus ersehen kann, dass kein verantwortlicher Politiker in diesen Staaten ernsthaft darüber nachdenkt, diese Entscheidung wieder rückgängig zu machen.

Aber auch jede speziell auf die Bundesrepublik Deutschland bezogene objektive Analyse muss eindeutig zu dem Ergebnis kommen, dass die Nachteile, die in der Beibehaltung der Wehrpflicht liegen, eventuelle Vorteile bei Weitem überwiegen. Dies gilt auch und gerade im Hinblick auf den gewichtigen Aspekt der Integration der Streitkräfte in eine demokratisch-pluralistische Gesellschaft, dem in der gesamten Debatte zu Recht ein hoher Stellenwert eingeräumt wird. Die Abschaffung der Wehrpflicht, zu der im Übrigen heute in Deutschland gerade einmal noch 15 Prozent der männlichen Bevölkerung herangezogen werden, muss diesen Integrationsbestrebungen keineswegs abträglich sein - ganz im Gegenteil. ${ }^{5}$

Entscheidend in diesem Zusammenhang ist, dass man nicht jeden der in die Debatte einfließenden Gesichtspunkte einfach nur ungewichtet und gleichrangig nebeneinander stellen darf. Selbst wenn alle übrigen Argumente für eine Beibehaltung der Wehrpflicht sprächen (was mitnichten der Fall ist), so würden sie in ihrer Gesamtheit doch von einem einzigen, zentralen Aspekt überstrahlt werden: Nach einem vielfach zitierten Diktum des ehemaligen Bundespräsidenten Roman Herzog aus dem Jahr 1995 ist die Wehrpflicht nämlich „ein so tiefer Eingriff in die individuelle Freiheit des jungen Bürgers, dass ihn der demokratische Rechtsstaat nur fordern darf, wenn es die äußere Sicherheit des Staates wirklich gebietet. Sie ist also kein allgemeingültiges ewiges Prinzip, sondern sie ist auch abhängig von der konkreten Sicherheitslage. Ihre Beibehaltung, Aussetzung oder Abschaffung und ebenso die Dauer des Grundwehrdiens-

5 Eine Zusammenfassung der zentralen Argumente in der Wehrpflichtdebatte bei Jürgen Groß, Demokratische Streitkräfte, Baden-Baden 2005, S. 59ff; die aktuelle Zahlenangabe bei Bernzen/Bertram in diesem Heft. tes müssen sicherheitspolitisch begründet werden können."6 Mit anderen Worten: Die Wehrpflicht muss für die Sicherheit der Bundesrepublik Deutschland zwingend erforderlich und ohne verantwortbare Alternative sein. Daran konnte man schon zuzeiten der Weizsäcker-Kommission eigentlich nicht mehr ernsthaft glauben - und heute erst recht nicht. (Heute ist die Frage nicht mehr, ob die Sicherheit unseres Landes von der allgemeinen Wehrpflicht abhängt, sondern ob sie vielleicht sogar schon von der Existenz nationaler Streitkräfte weitestgehend unabhängig geworden ist. ${ }^{7}$ ) Bereits für sich allein genommen ist dies also eine völlig ausreichende Begründung dafür, um auch in Deutschland die Wehrpflicht abzuschaffen.

Eine Auswahlwehrpflicht ist also nicht in erster Linie deshalb abzulehnen, weil sie möglicherweise dem Gleichheitsgebot des Grundgesetzes widerspräche. Obwohl Gleichheit ohne jeden Zweifel ein sehr hoher menschlicher Wert ist, im Zweifelsfall sogar der allerhöchste, ${ }^{8}$ würde die Ablehnung aus einer solchen Begründung heraus ja letztlich, wie von den Befürwortern dieses Modells zu Recht angeführt wird, zu der in der Tat „unerträglichen Folgerung führen, das Gleichheitsgrundrecht des einzelnen Einberufenen durch einen ungerechtfertigten Eingriff in die Freiheitsrechte der Nicht-Einberufenen wahren zu wollen". 9

Vielmehr weist dieser letztgenannte Gesichtspunkt überhaupt erst auf den eigentlichen Kern des Problems hin: Die Auswahlwehrpflicht ist nämlich vor allem deshalb abzulehnen, weil sie einen anderen Grundwert, nämlich den der individuellen Freiheit, auch künftig, und zwar ganz ohne Not, immer noch bei vielen erheblich beeinträchtigen würde, selbst wenn die Zahl der Betroffenen in diesem Fall geringer wäre. Der Vorschlag, eine Auswahlwehrpflicht an die Stelle der derzeitigen Einberufungspraxis zu setzen, greift daher zu kurz. Die Wehrpflicht würde durch ein solches Auswahlverfahren nur verändert; es kommt darauf an, sie abzuschaffen.

6 Zit. n. Dieter S. Lutz, Die allgemeine Wehrpflicht: Militärisch überflüssig, verfassungsrechtlich bedenklich, finanziell belastend, in: Reinhard Mutz/Bruno Schoch/Friedhelm Solms (Hrsg.), Friedensgutachten 1997, Münster 1997, S. 185-198, hier S. 190

7 Vgl. Jürgen Groß, Demokratie, Sicherheit und Militär, in: Detlef Bald/HansGünter Fröhling/Jürgen Groß (Hrsg.), Bundeswehr im Krieg - wie kann die Innere Führung überleben? Hamburger Beiträge zur Friedensforschung und Sicherheitspolitik, Heft 153, Hamburg 2009, S. 46-59, hier S. 58.

8 Vgl. dazu auch Jürgen Groß, Philosophie der Gleichheit, Baden-Baden 2009. 9 Bernzen/Bertram in diesem Heft. 